\title{
Etnobotânica de plantas medicinais aromáticas: preparações e usos da flora local em cinco comunidades rurais localizadas na região do Baixo Tocantins, Pará, Brasil
}

\author{
Ethnobotany of aromatic medicinal plants: preparations and uses of local flora in five rural \\ communities located in the region of Baixo Tocantins, Pará, Brazil \\ Etnobotánica de plantas medicinales aromáticas: preparaciones y usos de la flora local en cinco \\ comunidades rurales ubicadas en la región del Baixo Tocantins, Pará, Brasil
}

\section{Resumo}

As plantas medicinais constituem uma fonte primária de recursos naturais, utilizadas no tratamento de afecções pela população de comunidades ribeirinhas localizadas na Amazônia brasileira. Dessa forma, objetivou-se fazer um levantamento etnobotânico das plantas medicinais aromáticas, bem como, as indicações, as partes das plantas mais utilizadas, os modos de preparo dos remédios caseiros e verificar os valores de concordância de uso principal das espécies utilizadas pelos moradores de cinco comunidades rurais localizadas na região do Baixo Tocantins, Pará, Brasil. A seleção dos colaboradores se deu através da metodologia "bola de neve". Nessa pesquisa foram entrevistados 78 participantes na faixa etária de 35 a 82 anos, com predomínio do gênero feminino, e as plantas com predomínio de uso foram erva cidreira, comida de jabuti e priprioca. No total foram coletadas e identificadas 29 espécies, incluídas em 23 gêneros e 11 famílias, sendo Lamiaceae a mais representativa. Nessas comunidades as plantas medicinais aromáticas, além de tratar doenças, fazem parte da cultura, dos costumes e fortalecem as relações sociais e de convivência.

Palavras-chave: Flora aromática; Saberes populares; Produtos naturais amazônicos. 


\begin{abstract}
Medicinal plants are a primary source of natural resources, used to treat conditions by the population of riverside communities located in the Brazilian Amazon. Thus, the objective was to carry out an ethnobotanical survey of the aromatic medicinal plants, as well as the indications, the parts of the most used plants, the ways of preparing home remedies and to verify the agreement values for the main use of the species used by residents of five rural communities located in the Baixo Tocantins region, Pará, Brazil. The selection of employees took place through the "snowball" methodology. In this research 78 participants were interviewed in the age group from 35 to 82 years old, with a predominance of the female gender, and the plants with a predominance of use were lemongrass, tortoise and priprioca food. In total 29 species were collected and identified, included in 23 genera and 11 families, with Lamiaceae being the most representative. In these communities, aromatic medicinal plants, in addition to treating diseases, are part of culture, customs and strengthen social and coexistence relationships.
\end{abstract}

Keywords: Aromatic flora; Popular knowledge, Amazonian natural products.

\title{
Resumen
}

Las plantas medicinales son una fuente primaria de recursos naturales, utilizados para tratar las condiciones de la población de las comunidades ribereñas ubicadas en la Amazonía brasileña. Así, el objetivo fue realizar un relevamiento etnobotánico de las plantas aromáticas medicinales, así como las indicaciones, las partes de las plantas más utilizadas, las formas de preparar remedios caseros y verificar los valores de concordancia de uso principal de las especies utilizadas por los pobladores de cinco comunidades rurales ubicadas en la región del Baixo Tocantins, Pará, Brasil. La selección de empleados se realizó mediante la metodología "bola de nieve". En esta investigación se entrevistaron 78 participantes en el grupo etario de 35 a 82 años, con predominio del género femenino, y las plantas con predominio de uso fueron limoncillo, tortuga y priprioca. En total se recolectaron e identificaron 29 especies, incluidas en 23 géneros y 11 familias, siendo Lamiaceae la más representativa. En estas comunidades, las plantas medicinales aromáticas, además de tratar enfermedades, forman parte de la cultura, las costumbres y fortalecen las relaciones sociales y de convivencia.

Palabras clave: Flora aromática; Conocimiento popular; Productos naturales amazónicos.

\section{Introdução}

As plantas medicinais aromáticas (PMA) possuem aroma característico devido ao óleo essencial contido em uma ou mais partes e encontram-se distribuídas em diversas famílias botânicas, entre as quais podem ser destacadas: Apiaceae, Asteraceae, Cupressaceae, Fabaceae, Hypericaceae, Lamiaceae, Lauraceae, Myrtaceae, Pinaceae, Piperaceae, Poaceae, Rutaceae e Zingiberaceae (Noriega et al., 2019).

Na Amazônia brasileira muitas espécies de PMA já foram estudadas, como Ontrichardia linifera Schott (Araceae), Myrcia eximia DC. (Myrtaceae), Piper aduncum L. (Piperaceae), Lippia thymoides Mart. \& Schauer (Verbanaceae), Piper divaricatum G. Mey (Piperaceae) (Batista et al., 2019; Oliveira et al., 2019a; Silva et al., 2019a; Vilhena et al., 2019; Ferreira et al., 2020). Nas comunidades amazônicas, principalmente aquelas que se encontram localizadas distantes dos grandes centros médicos e de diagnósticos, esses vegetais constituem a fonte primária de recursos para cuidar da saúde ou curar de diversas afecções (Santos et al., 2019).

Segundo Santos et al. (2019), a indicação de uma PMA, dependendo da cultura e dos costumes de uma comunidade, segue uma ritualística que consiste em relatar os casos em que as formulações funcionaram; explicar as etapas do preparo; destacar as fases mais importantes do tratamento que devem ser cumpridas e as implicações em não cumpri-las. Nesse contexto, de acordo com Meyer et al. (2012), a etnobotânica é fundamental para registrar esses conhecimentos empíricos e valorizar o patrimônio cultural dessas comunidades, pois esses saberes são construídos com base nas relações com a natureza na qual estão incluídos.

Estudos etnobotânicos podem ser valiosos para registrar as espécies usadas pelas comunidades e testadas empiricamente ao longo de gerações, desde que respeitando a legislação. Além disso, podem contribuir para descoberta de novas espécies, manejo e preservação da flora medicinal. Sendo assim, as formas de usos das plantas medicinais na medicina popular envolvem as famílias e as comunidades, dentro de uma cadeia de trocas de saberes que são passados, oralmente, 
destacando o papel das mulheres com mais idade que atuam ativamente na manutenção da cultura e dos conhecimentos relativos às plantas medicinais (Pereira \& Coelho 2017).

A utilização em larga escala das PMA pode ser devido as múltiplas propriedades terapêuticas atribuídas, principalmente, aos óleos essenciais. Os quais são uma mistura de produtos químicos hidrofóbicos extraídos das plantas, altamente concentrados e voláteis (Silva et al., 2019a; Vilhena et al., 2019; Ferreira et al., 2020). Nesse contexto, esse estudo objetivou fazer o levantamento da flora medicinal e aromática utilizadas pelos moradores de cinco comunidades rurais, bem como, as indicações, as partes das plantas mais utilizadas, os modos de preparo dos remédios caseiros e verificar os valores de concordância de uso principal das espécies.

\section{Metodologia}

\subsection{Local da pesquisa}

O levantamento etnobotânico das PMA incluídas nessa pesquisa foi desenvolvido em cinco comunidades rurais: Ilha Trambioca (Barcarena), Rios Doce e Prata (Abaetetuba), Mamangal (Igarapé-Miri) e Nossa Senhora do Livramento (Acará). Esses municípios encontram-se localizados no Estado do Pará, região Norte do Brasil, Baixo Tocantins (Figura 1).

Figura 1. Mapa do estado Pará e localização dos municípios Abaetetuba, Acará, Barcarena e de Igarapé-Miri.

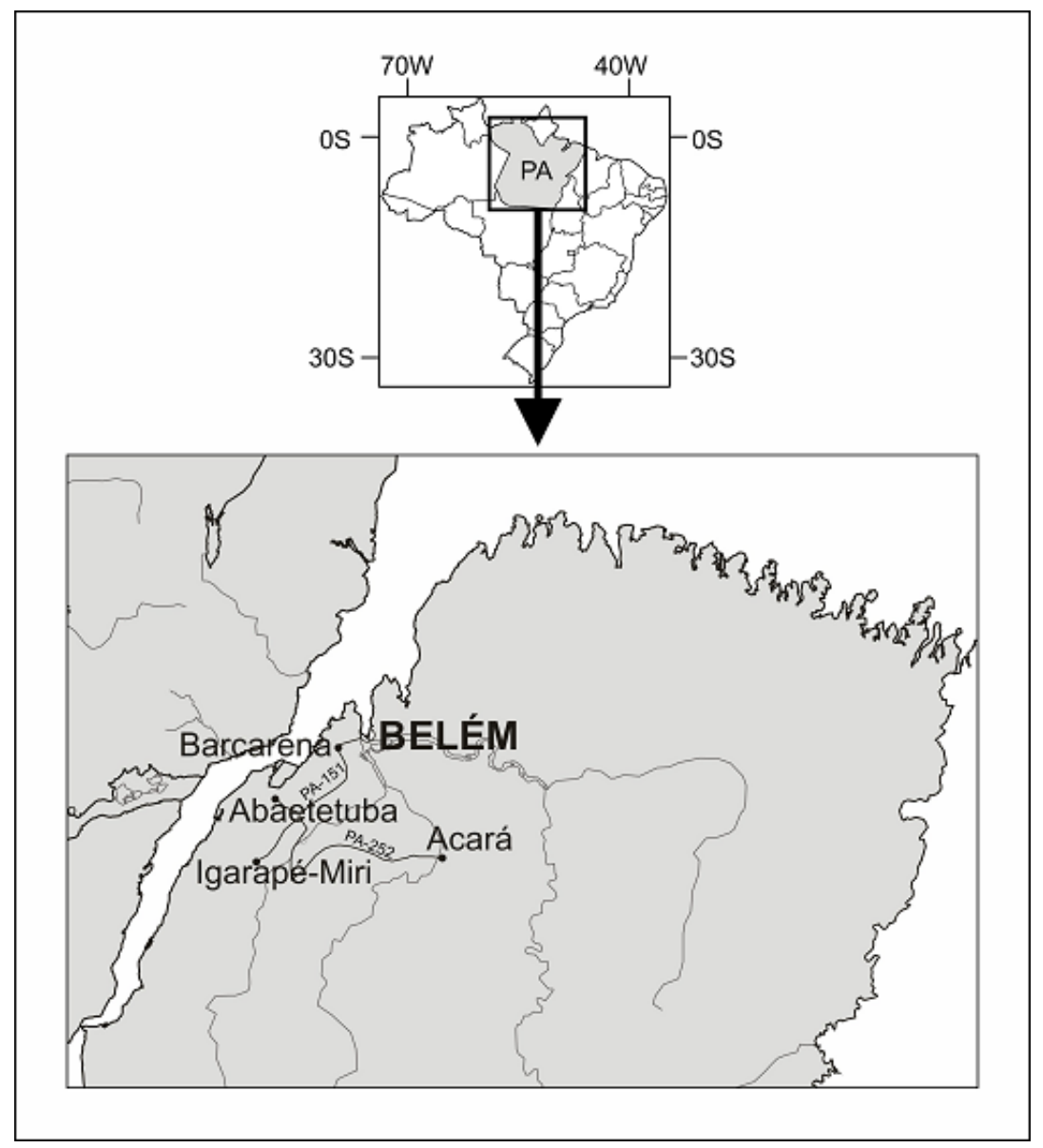

Fonte: Autores (2020).

A Ilha Trambioca está situada no município de Barcarena (1³1'8” Sul e 48³7'1" Oeste), abrangendo uma área de 8.486 hectares banhada pelos rios Mucuruçá, furo do Arrozal, rio Carnapijó e baía do Marajó (Souza \& Lisboa, 2005). As comunidades distribuídas ao longo da ilha, geralmente, ocupam ambientes ribeirinhos e de terra firme. A principal via de 
acesso à ilha é feita através da travessia de balsa e pequenas embarcações do rio Mucuruçá, em frente à sede municipal e as atividades econômicas mais importantes são a pesca artesanal, agricultura familiar e o extrativismo vegetal (Simonian, 2004).

As comunidades Rios Doce e da Prata encontram-se localizadas nas ilhas Sapucajuba e Rio da Prata, respectivamente, município de Abaetetuba (01 ${ }^{\circ} 43^{\prime}$ 05" Sul e 48 52' 57" Oeste). As fontes de renda nas comunidades Rios Doce e da Prata provêm da pesca artesanal, da colheita do açaí (Euterpe oleracea Mart, Arecaceae) e da fabricação de matapi (instrumento de pesca utilizado na captura de camarões) (Leal et al., 2019).

A comunidade Mamangal situa-se as margens do Rio Mamangal, no município de Igarapé-Miri (158’37” Sul e 4857'34” Oeste) (Pinto et al., 2014). A Comunidade de Nossa Senhora do Livramento, está localizada na Rodovia PA 252, Km 05, município de Acará (157’37” Sul e 48¹1'47” Oeste). A Comunidade conta com 18 famílias, tendo como principal fonte de renda a venda da farinha de mandioca. Os moradores que não estão ligados à plantação de mandioca são servidores públicos ou aposentados ou trabalham nas plantações dendê (Batista et al., 2020; Mesquita et al., 2020). A obtenção das informações etnobotânicas nas cinco comunidades rurais ocorreu entre os anos de 2018 e 2019 (Tabela 1).

Tabela 1. Localidades e números de participantes em cada uma das cinco comunidades rurais no estado do Pará, Brasil.

\begin{tabular}{|c|c|c|}
\hline Cidades & Comunidades & $\begin{array}{l}\text { Número de } \\
\text { participantes }\end{array}$ \\
\hline \multirow[t]{2}{*}{ Abaetetuba } & Rio Doce & 25 \\
\hline & Rio Prata & 10 \\
\hline \multirow[t]{2}{*}{ Barcarena } & Ilha & 11 \\
\hline & Trambioca & \\
\hline \multirow[t]{2}{*}{ Acará } & Nossa & 12 \\
\hline & $\begin{array}{l}\text { Senhora do } \\
\text { Livramento }\end{array}$ & \\
\hline $\begin{array}{l}\text { Igarapé- } \\
\text { Miri }\end{array}$ & Mamangal & 20 \\
\hline Total & 5 & 78 \\
\hline
\end{tabular}

\subsection{Amostragem e coleta dos dados}

A seleção dos colaboradores se deu através da metodologia "bola de neve" (snowball sampling) (Albuquerque et al., 2010), na qual os líderes comunitários indicaram os primeiros informantes e estes, recomendaram os próximos, repetindo-se o processo a partir de novos sujeitos incluídos.

A pesquisa de campo foi realizada em três etapas: primeiramente visitou-se os líderes comunitários que indicaram os possíveis participantes; a segunda fase consistiu em aplicar os questionários, explicar os objetivos da pesquisa, a leitura do Termo de Consentimento Livre e Esclarecido (TCLE) pelos participantes e a confirmação do consentimento em participar da pesquisa; na terceira e última adotou-se os procedimentos de coleta das amostras. Durante a coleta os interlocutores foram indagados sobre as plantas aromáticas e aquelas que se enquadravam nessa categoria receberam uma etiqueta diferenciada. A técnica da lista livre foi adotada, a qual consiste em citar componentes de um determinado domínio cultural na ordem que o participante lembrar, representando o conhecimento local e sua variação na comunidade (Quilan, 2005).

A pesquisa fez parte do projeto "Etnobotânica e o conhecimento regional das plantas medicinais das famílias Apocynaceae, Moraceae, Meliaceae e Fabaceae das microrregiões de Cametá e Tomé Açu, Pará, Brasil” e encontra-se cadastrado no Sistema Nacional de Gestão do Patrimônio Genético e do Conhecimento Tradicional Associado (SisGen), sob o número A512ABA. 


\subsection{Identificação botânica}

As coletas dos espécimes vegetais e as visitas as comunidades Ilha Trambioca, Rios Doce e Prata, Mamangal e Nossa Senhora do Livramento foram realizadas por meio de turnês guiadas. A herborização seguiu técnicas descritas por Ming (1996) e sempre que possível, coletou-se amostras férteis e fez-se registro fotográfico das plantas medicinais aromáticas incluídas na amostra. A identificação das espécies teve como base a literatura especializada e consultas as plataformas: Flora do Brasil 2020 e Trópicos do Missouri Botanical Garden. As exsicatas encontram-se catalogadas e incorporadas à coleção biológica do Herbário do Instituto Federal do Pará, Campus Abaetetuba, Pará, Brasil.

\subsection{Análise dos dados}

A Porcentagem de Concordância de Uso Principal (CUP) indicou a importância relativa das plantas utilizadas nas comunidades Ilha Trambioca, Rios Doce e Prata, Mamangal e Nossa Senhora do Livramento. Para evitar distorções entre espécies citadas por muitos participantes e aquelas citadas por poucos, o valor da CUP encontrado foi multiplicado por um fator de correção (FC), para cada espécie. Para a variável CUPc, foram considerados as plantas citadas por 10 ou mais colaboradores (Amorozo \& Gély, 1998). A CUP para cada espécie, foi calculada da seguinte forma:

$\mathrm{CUP}_{=} \quad$ Número de informantes que citaram usos principais $\mathrm{X} 100$

Número de informantes que citaram uso da espécie

O valor da CUP encontrado foi multiplicado por um fator de correção (FC), conforme a fórmula:

$\mathrm{FC}=$ Número de informantes que citaram a espécie $\mathrm{X} 100$

Número de informantes que citaram a espécie mais citada

A CUP corrigida é dada então:

$$
\mathrm{CUPc}=\mathrm{CUP} \times \mathrm{FC}
$$

\section{Resultados e Discussão}

Essa pesquisa contou com 78 participantes, sendo 72 do gênero feminino e 6 do masculino. A faixa etária variou entre 35 a 82 anos. Dos participantes, 85\% são alfabetizados, 96\% relataram ter nascido e morar na mesma comunidade até a participação nesse estudo. Os moradores dessas comunidades, incluídos nesse levantamento etnobotânico, informaram não cobrar pelo ato de benzer, fazer a indicação das ervas ou pela formulação caseira já pronta.

O predomínio da presença das mulheres e na faixa etária acima dos 40 anos de idade foram relatados em outros estudos etnobotânicos (Santos et al., 2019; Mesquita t al., 2020). Entretanto, segundo os colaboradores dessa pesquisa, as PMA nas cinco comunidades não são exploradas com objetivo de gerar renda, diferentemente da exploração de outras espécies como as andirobeiras (Carapa guianensis Aubl, Meliaceae) (De Sousa et al. 2019a) e os amapazeiros (Parahancornia fasciculata (Poir) Benoist, Apocynaceae) (De Sousa et al., 2019b).

Nesse estudo, construiu-se uma lista livre com 300 etnoespécies de plantas medicinais, das quais 70 foram identificadas como sendo aromáticas. Dessas 70 plantas, 29 espécimes foram coletados e identificadas, pertencendo a 11 famílias, 23 gêneros e 29 espécies. Todas as espécies foram confirmadas como sendo aromáticas, demonstrando concordância entre o conhecimento popular e ciência acadêmica (Quadro 1). 
Quadro 1. PMA usadas na medicina popular citadas por pelo menos 10 participantes em cinco comunidades rurais na Região do Baixo Tocantins, Pará, Brasil.

\begin{tabular}{|c|c|c|c|c|}
\hline Etnoespécies & Família/nome científico & Indicações & Preparações & Constituintes majoritários \\
\hline $\begin{array}{l}\text { Alecrim de } \\
\text { planta }\end{array}$ & $\begin{array}{l}\text { Lamiaceae } \\
\text { Rosmarinus officinalis } \\
\text { L. }\end{array}$ & $\begin{array}{l}\text { Infecção } \\
\text { urinária. }\end{array}$ & $\begin{array}{l}\text { Chá das folhas misturado } \\
\text { com quebra pedra. Tomar } \\
\text { três vezes ao dia, por } 10 \\
\text { dias. }\end{array}$ & $\begin{array}{l}\alpha \text {-Pineno, 8-cineol, canfeno, } \\
\text { verbenona (Silvestre et al., } \\
\text { 2019). }\end{array}$ \\
\hline Alfavaca & $\begin{array}{l}\text { Lamiaceae } \\
\text { Ocimum basilicum } \mathrm{L} \text {. }\end{array}$ & $\begin{array}{l}\text { Sinusite, } \\
\text { espirros e } \\
\text { nariz entupido. }\end{array}$ & $\begin{array}{l}\text { Ferver as folhas e cheirar o } \\
\text { vapor. Depois que esfriar } \\
\text { molhar a cabeça. }\end{array}$ & $\begin{array}{l}\text { Linalool, eugenol (Filho et } \\
\text { al., 2006). }\end{array}$ \\
\hline $\begin{array}{l}\text { Anador de } \\
\text { planta }\end{array}$ & $\begin{array}{l}\text { Lamiaceae } \\
\text { Plectranthus barbatus } \\
\text { Adrews }\end{array}$ & $\begin{array}{l}\text { Dores em } \\
\text { geral. }\end{array}$ & Chá das folhas. & $\begin{array}{l}\text { Eugenol, timol, carvacrol } \\
\text { (Rodrigues et al., 2013). }\end{array}$ \\
\hline $\begin{array}{l}\text { Anador } \\
\text { quintal }\end{array}$ & $\begin{array}{l}\text { Lamiaceae } \\
\text { Plectranthus neochilus } \mathrm{S} \\
\text { chltr }\end{array}$ & $\begin{array}{l}\text { (1) Dores em } \\
\text { geral; } \\
\text { (2) Tosse } \\
\text { produtiva. }\end{array}$ & $\begin{array}{l}\text { (1) Chá das folhas. (2) } \\
\text { Aquecer as folhas com } \\
\text { açúcar até formar uma calda } \\
\text { grossa. Depois de esfriar } \\
\text { tomar uma colher junto com } \\
\text { as refeições. }\end{array}$ & 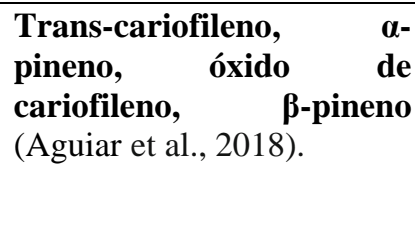 \\
\hline Arnica & $\begin{array}{l}\text { Asteraceae } \\
\text { Solidago chilensis } \\
\text { Meyen }\end{array}$ & $\begin{array}{l}\text { Inflamação e } \\
\text { batida no } \\
\text { corpo (baque). }\end{array}$ & $\begin{array}{l}\text { Colocar as folhas dentro de } \\
\text { um vidro com óleo de } \\
\text { andiroba, deixar por cinco } \\
\text { dias e passar no machucado } \\
\text { ou no local inflamado. }\end{array}$ & $\begin{array}{l}\text { Limoneno, } \beta \text {-elerneno, } \gamma_{-} \\
\text {cadineno, pumilóxido (Vila } \\
\text { et al., 2001). }\end{array}$ \\
\hline Arruda & $\begin{array}{l}\text { Rutaceae } \\
\text { Ruta graveolens L. }\end{array}$ & $\begin{array}{l}\text { (1) Febre de } \\
\text { criança. } \\
\text { (2) Mau } \\
\text { olhado. } \\
\text { (3) Dor de } \\
\text { cabeça. }\end{array}$ & $\begin{array}{l}\text { (1) Socar as folhas no pilão, } \\
\text { tirar o sumo e misturar com } \\
\text { leite materno e dar para o } \\
\text { bebê tomar. (2) Tomar } \\
\text { banho com o chá das folhas. } \\
\text { (3) Socar as folhas no pilão } \\
\text { e colocar na cachaça. Modo } \\
\text { de usar: umedecer um pano } \\
\text { branco e colocar na cabeça. }\end{array}$ & 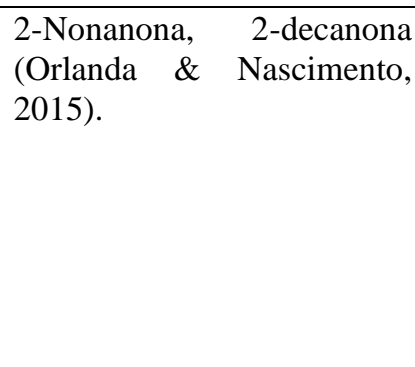 \\
\hline $\begin{array}{l}\text { Boldo do } \\
\text { china }\end{array}$ & $\begin{array}{l}\text { Lamiaceae } \\
\text { Plectranthus ornatus } \\
\text { Codd }\end{array}$ & $\begin{array}{l}\text { Problemas no } \\
\text { fígado e dor } \\
\text { no estômago. }\end{array}$ & Chá das folhas. & $\begin{array}{l}\text { Cariofileno, } \beta \text {-cariofileno, } \\
\text { terpinen-4-ol (Alves et al., } \\
\text { 2018). }\end{array}$ \\
\hline Boldo planta & $\begin{array}{l}\text { Asteraceae } \\
\text { Vernonia condensata } \\
\text { Baker }\end{array}$ & $\begin{array}{l}\text { Dor de } \\
\text { estômago. }\end{array}$ & Chá das folhas. & $\begin{array}{l}\beta \text {-cariofileno, } \gamma \text {-muuroleno, } \\
\text { mirceno, } 2 \text { - } \delta \text {-careno, } 1,8- \\
\text { cineol (Lopes et al., 2019). }\end{array}$ \\
\hline Borboleta & $\begin{array}{l}\text { Zingiberaceae } \\
\text { Hedychium coronarium } \\
\text { J. Koenig }\end{array}$ & $\begin{array}{l}\text { Baixar } \\
\text { albumina. }\end{array}$ & $\begin{array}{l}\text { Chá das folhas ou das } \\
\text { raízes. }\end{array}$ & $\begin{array}{l}\beta \text {-pineno, 1.8-cineol, } \alpha \text { - } \\
\text { pineno (Noriega et al., } \\
\text { 2019). }\end{array}$ \\
\hline Canela & $\begin{array}{l}\text { Lauraceae } \\
\text { Cinnamomum verum } \mathrm{J} . \\
\text { Presl }\end{array}$ & Calmante & Chá das folhas. & $\begin{array}{l}\text { Linalool, } \quad \beta \text {-bisabolene, } \\
\text { bicyclogermacrene } \quad \text { (Chinh } \\
\text { et al., 2017). }\end{array}$ \\
\hline $\begin{array}{l}\text { Capim } \\
\text { citronela }\end{array}$ & $\begin{array}{l}\text { Poaceae } \\
\text { Cymbopogon } \\
\text { winterianus Jowitt ex } \\
\text { Bor }\end{array}$ & $\begin{array}{l}\text { Repelente } \\
\text { contra } \\
\text { mosquitos. }\end{array}$ & $\begin{array}{l}\text { Chá das folhas para passar } \\
\text { na pele (bem forte). }\end{array}$ & $\begin{array}{ll}\text { Citronelal, } & \text { citronelol, } \\
\text { geraniol, elemol } & \text { (Chooluck } \\
\text { et al., 2019). } & \end{array}$ \\
\hline
\end{tabular}




\begin{tabular}{|c|c|c|c|c|}
\hline Capim limão & $\begin{array}{l}\text { Poaceae } \\
\text { Cymbopogon flexuosus } \\
\text { (Nees) Stapf }\end{array}$ & Pressão alta. & Chá das folhas. & $\begin{array}{l}\text { Geranial, neral, geranial (Da } \\
\text { Silva et al., 2018). }\end{array}$ \\
\hline Capim santo & $\begin{array}{l}\text { Poaceae } \\
\text { Cymbopogon citratus } \\
\text { (L.) Stapf }\end{array}$ & Febre. & $\begin{array}{l}\text { Banho: cozinhar as folhas e } \\
\text { depois de esfriar, } \\
\text { acrescentar álcool. Molhar } \\
\text { todo corpo até a febre } \\
\text { baixar. }\end{array}$ & $\begin{array}{l}\text { Geranial, neral, } \beta \text {-mircene } \\
\text { (Da Silva et al., 2018). }\end{array}$ \\
\hline $\begin{array}{l}\text { Cidreira - Erva } \\
\text { cidreira }\end{array}$ & $\begin{array}{l}\text { Verbenaceae } \\
\text { Lippia alba (Mill.) N. E. } \\
\text { Br. ex Britton \& P. } \\
\text { Wilson }\end{array}$ & $\begin{array}{l}\text { Baixar } \\
\text { pressão. }\end{array}$ & Chá folhas. & $\begin{array}{l}\text { Linalol, trans-ocimenona, } \\
\text { óxido de } \quad \text { cariofileno } \\
\text { (Arruda et al., 2019). }\end{array}$ \\
\hline $\begin{array}{l}\text { Cidreira- } \\
\text { Camilitana }\end{array}$ & $\begin{array}{l}\text { Lamiaceae } \\
\text { Melissa officinalis L. }\end{array}$ & $\begin{array}{l}\text { Dor no } \\
\text { estômago. }\end{array}$ & $\begin{array}{l}\text { Chá das folhas. Tomar uma } \\
\text { vez ao dia até a dor passar. }\end{array}$ & $\begin{array}{l}\text { Geranial, } \\
\text { cariofileno } \\
\text { 2020). }\end{array}$ \\
\hline $\begin{array}{l}\text { Comida de } \\
\text { jabuti }\end{array}$ & $\begin{array}{l}\text { Piperaceae } \\
\text { Peperomia pellucida } \\
\text { (L.) Kunth }\end{array}$ & $\begin{array}{l}\text { Frieiras } \\
\text { (coceira nas } \\
\text { mãos e nos } \\
\text { pés). }\end{array}$ & $\begin{array}{l}\text { Socar as folhas no pilão, } \\
\text { tirar o sumo e colocar } \\
\text { cachaça ou amoníaco. } \\
\text { Colocar numa vasilha e } \\
\text { deixar as mãos ou os pés de } \\
\text { molho. }\end{array}$ & $\begin{array}{l}\text { Linalol, d-limoneno, } \beta- \\
\text { cariofileno (Okoh et al., } \\
\text { 2017). }\end{array}$ \\
\hline $\begin{array}{l}\text { Cordão de são } \\
\text { Francisco }\end{array}$ & $\begin{array}{l}\text { Lamiaceae } \\
\text { Leonotis nepetifolia (L.) } \\
\text { R. Br. }\end{array}$ & $\begin{array}{l}\text { Inflamação em } \\
\text { geral. }\end{array}$ & Chá das folhas. & $\begin{array}{l}\text { Germacreno-D, } \beta- \\
\text { cariofileno, } \quad \text { B-elemeno } \\
\text { (Emmanuel et al., 2018). }\end{array}$ \\
\hline Gengibre & $\begin{array}{l}\text { Zingiberaceae } \\
\text { Zingiber officinale } \\
\text { Roscoe }\end{array}$ & $\begin{array}{l}\text { (1) Dores nas } \\
\text { juntas. } \\
\text { (2) Tosse. }\end{array}$ & $\begin{array}{l}\text { (1) Triturar as batatas no } \\
\text { pilão e colocar no álcool. } \\
\text { Depois que estiver bem } \\
\text { forte, colocar andiroba e } \\
\text { banha de galinha. Passar } \\
\text { nos locais que sente as } \\
\text { dores. (2) Chá da batata } \\
\text { com caatinga de mulata, } \\
\text { hortelã grande e e } \\
\text { panamenha. Acrescentar } \\
\text { mel de abelha ou leite } \\
\text { condensado e tomar três } \\
\text { vezes ao dia até a tosse } \\
\text { passar. }\end{array}$ & $\begin{array}{l}\text { (-)-Zingibereno, 8-dieno, p- } \\
\text { mentha-1 (Oliveira et al., } \\
\text { 2019b). }\end{array}$ \\
\hline Hortelã verde & $\begin{array}{l}\text { Lamiaceae } \\
\text { Mentha spicata L. }\end{array}$ & $\begin{array}{l}\text { (1) Dores no } \\
\text { estômago e } \\
\text { intestino. (2) } \\
\text { Gripe com } \\
\text { tosse (chiado } \\
\text { no peito). }\end{array}$ & $\begin{array}{l}\text { (1) Chá das folhas. (2) } \\
\text { Xarope: aquecer as folhas } \\
\text { com açúcar até formar uma } \\
\text { calda de cor bem escura. } \\
\text { Tomar uma colher depois } \\
\text { das refeições. }\end{array}$ & $\begin{array}{l}\text { Carvone, limonene, } 1,8- \\
\text { cineol (Ali-Shtayeh et al., } \\
\text { 2019). }\end{array}$ \\
\hline $\begin{array}{l}\text { Majirona da } \\
\text { angola }\end{array}$ & $\begin{array}{l}\text { Verbenaceae } \\
\text { Lippia thymoides Mart. } \\
\text { \& Schauer }\end{array}$ & $\begin{array}{l}\text { (1) Mau } \\
\text { olhado, dor de } \\
\text { cabeça e falta } \\
\text { de ânimo. (2) } \\
\text { Derrame. }\end{array}$ & $\begin{array}{l}\text { (1) Banho: ferver as folhas e } \\
\text { depois de esfriar, molhar } \\
\text { todo corpo sempre a noite } \\
\text { antes de dormir por sete } \\
\text { dias. (2) Moer as folhas até } \\
\text { ficar bem úmida. Aquecer e } \\
\text { fazer massagem nas partes } \\
\text { do corpo afetada. }\end{array}$ & $\begin{array}{l}\text { Timol, acetato de timol, } \beta- \\
\text { cariofileno (Silva et al., } \\
\text { 2019a). }\end{array}$ \\
\hline Malvarisco & $\begin{array}{l}\text { Piperaceae } \\
\text { Piper umbellatum L. }\end{array}$ & $\begin{array}{l}\text { Inchaço das } \\
\text { juntas. }\end{array}$ & $\begin{array}{l}\text { Aquecer as folhas junto com } \\
\text { óleo de andiroba ou banha } \\
\text { de galinha e cobrir as partes } \\
\text { inchadas e doloridas. } \\
\text { Repetir várias vezes ao dia }\end{array}$ & $\begin{array}{l}\text { Piperitona, dill apoiole, } \\
\text { myrcene, } \rho \text {-mentha-1 (7), 8- } \\
\text { dieno, eudesmol (Dorneles } \\
\text { et al., 2019). }\end{array}$ \\
\hline
\end{tabular}


Research, Society and Development, v. 10, n. 1, e9510111284, 2021

(CC BY 4.0) | ISSN 2525-3409 | DOI: http://dx.doi.org/10.33448/rsd-v10i1.11284

\begin{tabular}{|c|c|c|c|c|}
\hline & & & até ficar sarado. & \\
\hline Mucuracaá & $\begin{array}{l}\text { Petiveriaceae } \\
\text { Petiveria alliacea } \mathrm{L} \text {. }\end{array}$ & Dor de dente. & $\begin{array}{l}\text { Colocar o sumo das folhas } \\
\text { sobre o local da dor. }\end{array}$ & $\begin{array}{l}\begin{array}{l}\text { Dimetilsufeto, } \\
\text { dietilsufeto } \\
\text { 2019). }\end{array} \\
\text { (Alves et al., }\end{array}$ \\
\hline Óleo elétrico & $\begin{array}{l}\text { Piperaceae } \\
\text { Piper callosum Ruiz \& } \\
\text { Pav. }\end{array}$ & $\begin{array}{l}\text { Prisão de } \\
\text { ventre. }\end{array}$ & $\begin{array}{l}\text { Chá das folhas juntamente } \\
\text { com alho e cebola roxa. }\end{array}$ & $\begin{array}{l}\text { Safrole, metyleugenol, } \\
\text { elemicina (Silva et al., } \\
\text { 2016). }\end{array}$ \\
\hline $\begin{array}{l}\text { Pataqueira } \\
\text { cheirosa }\end{array}$ & $\begin{array}{l}\text { Plantaginaceae } \\
\text { Conobea scoparioides } \\
\text { (Cham. \& Schltdl.) } \\
\text { Benth. }\end{array}$ & Mau olhado & $\begin{array}{l}\text { Banho: ferver as folhas e } \\
\text { acrescentar água benta. }\end{array}$ & 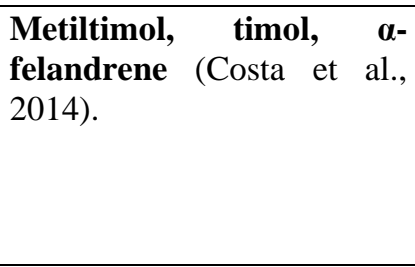 \\
\hline Pripioca & $\begin{array}{l}\text { Cyperaceae } \\
\text { Cyperus articulatus L. }\end{array}$ & Febre. & $\begin{array}{l}\text { Ralar o rizoma (batata) e } \\
\text { colocar no álcool. Depois de } \\
\text { apurado passar no corpo e } \\
\text { até a febre a baixar. }\end{array}$ & $\begin{array}{l}\text { Mustacone, } \\
\text { ciclocolorenona, } \alpha \text {-pineno } \\
\text { (Silva et al., 2019b). }\end{array}$ \\
\hline $\begin{array}{l}\text { Salva do } \\
\text { Marajó }\end{array}$ & $\begin{array}{l}\text { Lamiaceae } \\
\text { Hyptis subsect. crenata } \\
\text { Epling }\end{array}$ & $\begin{array}{l}\text { Cólica } \\
\text { menstrual e } \\
\text { diarreia. }\end{array}$ & Chá das folhas. & $\begin{array}{l}\text { p-Cimeno, 1,8-cineol, } \\
\text { borneol (Violante et al., } \\
\text { 2012). }\end{array}$ \\
\hline Terramicina & $\begin{array}{l}\text { Amaranthaceae } \\
\text { Alternanthera brasiliana } \\
\text { (L.) Kuntze }\end{array}$ & $\begin{array}{l}\text { (1) } \\
\text { Inflamação. } \\
\text { (2) Ferimentos } \\
\text { na pele. }\end{array}$ & $\begin{array}{l}\text { (1) Chá das folhas. (2) } \\
\text { Colocar nos ferimentos o } \\
\text { sumo das folhas. }\end{array}$ & $\begin{array}{l}\text { Aristoleno, } 2,6 \text {-di-t-butil-4- } \\
\text { metilfenol, } \quad \gamma \text {-eudesmol } \\
\text { (Wakabayashi et al., 2010). }\end{array}$ \\
\hline Vick de planta & $\begin{array}{l}\text { Lamiaceae } \\
\text { Mentha arvensis } \\
\text { L. }\end{array}$ & $\begin{array}{l}\text { Garganta } \\
\text { inflamada. }\end{array}$ & $\begin{array}{l}\text { Chá das folhas para fazer o } \\
\text { gargarejo. }\end{array}$ & $\begin{array}{l}\text { Pulegone, mentilacetato, } \\
\text { mentona (Maurya et al., } \\
\text { 2019). }\end{array}$ \\
\hline Vindicá & $\begin{array}{l}\text { Zingiberaceae } \\
\text { Alpinia zerumbet B. L. } \\
\text { Burtt. \& R. M. Sm. }\end{array}$ & $\begin{array}{l}\text { Dor no } \\
\text { coração. }\end{array}$ & $\begin{array}{l}\text { Chá da flor misturado com } \\
\text { as flores de laranjeira e } \\
\text { folhas de coramina. }\end{array}$ & $\begin{array}{l}\text { Terpinen-4-ol, 1,8-cineol, } \\
\text { sabineno (Souza et al., } \\
2018 \text { ). }\end{array}$ \\
\hline
\end{tabular}

Fonte: Autores (2020).

As famílias mais representativas foram Lamiaceae (10 espécies), Piperaceae (3 espécies), Poaceae (3 espécies), Zingiberaceae (3 espécies) e Verbenaceae (2 espécies). Esses resultados corroboram com outros estudos etnobotânicos de PMA, os quais descreveram a família Lamiacaea ocupando uma posição de destaque em relação ao número de espécies (Pereira \& Coelho, 2017; Neri et al., 2018; Soares et al., 2019). A família Lamiaceae destaca-se, também, por apresentar grande diversidade de espécies de plantas medicinais rica em óleos essenciais, os quais possuem atividades antioxidante, bactericida, fungicida e inseticida (Lima \& Cardoso, 2007).

Em relação à origem das PMA incluídas nesta pesquisa, a maioria eram exóticas, representando 64\% e as demais nativas (36\%). Em relação aos usos terapêuticos, as espécies exóticas foram indicadas para tratamento ou cura de 14 categorias de doenças e as nativas 12 , sendo que todas as afecções caracterizadas pela presença de dores foram tratadas exclusivamente por PMA exóticas. Esses resultados estão de acordo com a hipótese da diversificação de Albuquerque (2006), a qual sugere que a incorporação de plantas medicinais exóticas na flora medicinal local tem como finalidade reforçar essa coletânea vegetal com espécies contendo compostos metabólitos secundários altamente bioativos. Posteriormente, Alencar et al. (2016) 
verificaram a consistência dessa hipótese, ao constatar que plantas exóticas incluindo árvores e ervas, continham grande quantidade de flavonoides e terpenos, enquanto o grupo nativo possuía maiores quantidades de triterpenos e alcaloides.

O chá das folhas, flores, raízes ou rizomas são as formas predominantes de preparo dos remédios caseiros utilizados pelos moradores das comunidades Ilha Trambioca, Rios Doce e Prata, Mamangal e Nossa Senhora do Livramento. O banho e o xarope também foram citados, sendo que essas formulações requerem ingredientes adicionais em relação aos chás. Com a finalidade de intensificar ou potencializar a ação terapêutica desses remédios ou melhorar o sabor, são adicionados açúcar, leite condensado, mel de abelha, leite materno, óleo de andiroba, banha de galinha, amoníaco, álcool, cachaça ou água benta, totalizando 26 maneiras de preparo.

Segundo os entrevistados, em algumas situações, as plantas medicinais constituem a única alternativa para aliviar os sintomas ou curar doenças, principalmente nas comunidades mais afastadas, pois apenas em uma dessas comunidades (Ilha Trambioca) possui um posto de saúde com atendimento diário de uma técnica de enfermagem e uma vez por semana por um médico. Entretanto, há apenas farmácias para comprar medicamentos na comunidade Mamangal e, como agravante, essas comunidades não contam com serviços regulares de coleta de lixo, distribuição de água tratada ou esgoto sanitário.

Nas comunidades da Amazônia brasileira, além de prevenir, aliviar os sintomas ou curar as doenças biológicas, as plantas também servem para cuidar dos males espirituais, como no ato de benzer ou ao acrescentar água benta nos remédios caseiros. O uso do pano branco nas aplicações dos remédios, não é só uma questão da higiene ou cuidados com a saúde, mas é também considerado uma forma de agradecer as divindades da floresta, aos espíritos de luz, santos ou aos orixás.

Os valores percentuais de Concordância corrigida quanto aos Usos Principais (CUPc) acima de $50 \%$ foram encontrados para as espécies erva cidreira, comida de jabuti e pripioca (Tabela 2). Esses valores de CUPc para cada espécie equivalem ao maior número de pessoas que relataram a mesma forma de uso para a mesma planta, sugerindo maior concordância de uso nas comunidades em estudo. Segundo Freitas et al. (2015) a aceitação de uso de plantas medicinais por uma comunidade ou várias comunidades resulta em maior segurança em relação a eficácia do uso proposto, o que sugere a utilização ou o conhecimento desses vegetais por muitos moradores dessas localidades.

Tabela 2. Porcentagem de concordância quanto ao (s) uso (s) principal (is) (espécies citadas por 10 ou mais pessoas).

\begin{tabular}{llll}
\hline Etnoespécies & Nome científico & Uso principal & CUPc \\
\hline Terramicina & Alternanthera brasiliana (L.) Kuntze & Inflamação & 36,36 \\
Pataqueira cheirosa & Conobea scoparioides Benth. & Mau olhado & 45,85 \\
Priprioca & Cyperus articulatus L. & Febre & $\mathbf{5 6 , 8 8}$ \\
Cidreira (erva cidreira) & Lippia alba (Mill.) & Pressão alta. & $\mathbf{7 2 , 4 0}$ \\
Marijona da angola & Lippia thymoides Mart. \& Schauer & Derrame & 48,00 \\
Comida de jabuti & Peperomia pellucida (L.) Kunth & Coceira no corpo & $\mathbf{6 3 , 1 2}$ \\
Óleo elétrico & Piper callosum Ruiz \& Pav. & Prisão de ventre & 37,50 \\
Malvarisco & Piper umbellatum L. & Inchaço das juntas & 40,02 \\
Arnica & Solidago chilensis Meyen & Inflamação & 40,00 \\
\hline
\end{tabular}

Fonte: Autores (2020).

O valor de CUPc também sugere que a Lippia alba (Mill.) foi a mais difundida nas comunidades estudadas, pois apenas na comunidade Rio da Prata não houve nenhuma referência ao nome desse vegetal. As propriedades medicinais atribuídas à Lippia alba (Mill.) podem ser devido ao óleo essencial extraído das folhas que possui como compostos majoritários linalol, transocimenina e cariofileno. Experimentalmente, o óleo essencial da espécie demostrou atividade antifúngica, principalmente contra Sclerotinia slerotiorum, uma espécie de fungo relacionada a doenças em plantas de soja (Arruda et al., 2019). 
A espécie Peperomia pellucida (L.) Kunth, conhecida popularmente como Comida de jabuti encontra-se distribuída por todo território brasileiro, sendo um arbusto de cor verde brilhante, folhas ovais alternadas e inflorescências terminais (Verna et al., 2014). O óleo essencial extraído das folhas apresentou atividade antibacteriana, antioxidante e é constituído majoritariamente, por linalol, d-limoneno e $\beta$-cariofileno (Mohamad et al., 2015; Okoh et al., 2017).

O termo priprioca refere-se a três espécies de plantas aromáticas (Cyperus articulatus L., Cyperus prolixus Kunth e Cyperus rotundus L.) e o rizoma é utilizado para extração de óleo essencial, de cor amarelo intenso, odor forte e agradável (Zoghbi et al., 2008). Na cultura popular é empregada para preparação de banhos de cheiro (Fonseca et al., 2018), como ritualística (Carmo et al., 2015) e no tratamento de doenças respiratórias (Mesquita \& Tavares-Martins, 2018). A espécie $C$. articulatus foi citada nas cinco comunidades, como um vegetal conhecido por ser antitérmico e curar mau olhado.

\section{Considerações Finais}

As plantas medicinais aromáticas fazem parte da cultura, dos costumes e contribuem para melhorar as condições de vida dos moradores dessas comunidades. Não são usadas como fonte de renda, mas permitem relações sociais de convivência e trocas de conhecimentos, contribuindo para o bem-estar da comunidade.

\section{Referências}

Aguiar, G. P., Lima, K. A., Severino, M. E., Groppo, M., Ambrósio, S. R., Crevelin, E. J. (2018). Antifungal activity of the essential oils of Plectranthus neochilus (Lamiaceae) and Tagetes erecta (Asteraceae) cultivated in Brazil. International Journal of Complementary \& Alternative Medicine, 11 (1), 31-35.

Albuquerque, U. P. (2006). Re-examining hypotheses concerning the use and knowledge of medicinal plants: a study in the vegetation of Caatinga vegetation of NE Brazil. Journal of Ethnobiology and Ethomedicine, 2, 30. 10.1186/1746-4269-2-30.

Albuquerque, U. P., Lucena, R. F. P., Lins Neto, E. M. F. Seleção dos participantes da pesquisa. In. Albuquerque, U. P., Lucena, R. F. P., Cunha, L. V. F. C. (Org.). Métodos e técnicas na pesquisa etnobiológica e etnoecológica. ( 41-64). NUPEEA, 2010.

Alencar, N. L., Araújo, T. A. S., Amorim, E. L. C., Albuquerque, U. P. (2016). The inclusion and selection of medicinal plants in traditional pharmacopoeias, evidence in support of the diversification hypothesis. Economic Botany, 64 (1), 68-79.10.1007/s12231-009-9104-5.

Ali-Shtayeh, M. S., Rana, M. J., Abu-Zaitoun, S. Y., Khasati, A., Kalbouneh, S. R. (2019). Biological properties and bioactive components of Mentha spicata L. essential Oil: focus on potential benefits in the treatment of obesity, Alzheimer's Disease, Dermatophytosis, and Drug-Resistant Infections. Evidence-Based Complementary and Alternative Medicine, 2019, 1-11. https://doi.org/10.1155/2019/3834265.

Alves, C. A., Rodrigues, E., Lago, J. H. G., Prado, C. M., Girardi, C. D. N., Hipólide, D. C. (2019). Petiveria alliacea, a plant used in Afro-Brasilian smoke rituals, triggers pulmonar inflammation in rats. Revista Brasileira de Farmacognosia, 29 (2019), 656-664. https://doi.org/10.1016/j.bjp.2019.06.005.

Alves, F. A. R., Morais, S. M., Sobrinho, A. C. N., Silva, I. N., Martins, C. G., Silva, A. A. S., Fontinelle, R. O. S. (2018). Chemical composition, antioxidant and antifungal activities of essential oils and extracts from Plectranthus spp. against dermatophytes fungi. Revista Brasileira Saúde Produção Animal, Salvador, 9 (1), 105-115. http://dx.doi.org/10.1590/S1519-99402018000100010.

Amorozo, M. C. M., \& Gély, A. (1988). Uso de plantas medicinais por caboclos do Baixo Amazonas, Barcarena, PA, Brasil. Boletim do Museu Paraense Emílio Goeldi. Ciências Naturais, 4 (1), 47-131. http://doi.org/10.46357/bcnaturais.v15i3.214.

Arruda, R. C. O., Victório, C. P., Boaretto, A. G., Carollo, C. A., Farias, C. S., Marchetti, C. R., Santos, R. J., Giannesi, G. C., Silva, D. B. (2019). Essential oil composition, antifungal activity and leaf anatomy of Lippia alba (Verbenaceae) from Brazilian Chaco. Journal of Medicinal Plants Research, 13 (4), 7988. 10.5897/JMPR2018.6700.

Batista, J. A. S., Barbosa, M. S., Silva, F. N. L., Oliveira, L. C., Sousa, R. L. (2020). Descrição social, econômico e ambiental de comunidades rurais no Acará, Pará, Brasil. Research, Society and Development, 9 (7), 1-24, e423974294. http://dx.doi.org/10.33448/rsd-v9i7.4294

Batista, R. J. R., Amarante, C. B., Botelho, A. S., Andrade, E. H. A., Nascimento, L. D. (2019). Constituintes voláteis da raiz e do rizoma de Montrichardia linifera (Arruda) Schott (Araceae). Boletim do Museu Paraense Emílio Goeldi. Ciências Naturais, $14 \quad$ (2), 197-207. http://doi.org/10.46357/bcnaturais.v15i3.214.

Carmo, T. N., Lucas, F. C. A., Lobato, G. J. M., \& Gurgel, E. S. C. (2015). Plantas medicinais e ritualísticas comercializadas na feira da 25 de Setembro, Belém, Pará. Enciclopédia Biosfera, Centro Científico Conhecer, Goiânia, 11 (21), 3440-3467. 10.18677/EnciBio_2020D45.

Chinh, H. V., Luong, N. X., Thin, D. B., Dai, D. N., Hoi, T. M., Ogunwande, I. A. (2017). Essential Oils Leaf of Cinnamomum glaucescens and Cinnamomum verum from Vietnam. American Journal of Plant Sciences, 8, 2712-2721. http://www.scirp.org/journal/ajps. 
Chooluck, K., Teeranachaideekul, V., Jintapattanakit, A., Lomarat, P., Phechkrajang, C. (2019). Repellency Effects of Essential Oils of Cymbopogon winterianus, Eucalyptus globulus, Citrus hystrix and their major Constituents against Adult German Cockroach (Blattella germanica Linnaeus (Blattaria: Blattellidae). Jordan Journal of Biological Sciences, 12 (4), 519-523. http://jjbs.hu.edu.jo/.

Costa, R. G., Faria, L. J. G., Gusmão, S. A. L., Silva, J. K. R., Andrade, E. H. A., Maia, J. G. S. (2014). Essential oil of Pataqueira (Conobea Scoparioides Benth.): from natural occurrence and produced by hydroponics. Advancs in Plants \& Agriculture Research, 1 (3), 00015. https://medcraveonline.com/APAR/

Da Silva, L. E., Gonçalves, M. V. S., Amaral, W., Quadros, D. A., Reis, R. A., Amaral, L. D. P., Huergo, L. F., Garcia, B. (2018). Chemical composition and antibacterial activity of Cymbopogon citratus and Cymbopogon flexuosus essential oils. Ciência e Natura, Santa Maria, 40, (e2). 10.5902/2179460X27569.

De Sousa, R. L., Costa, J. F. S., Costa, J. M., Pereira, M. G. S., Cordeiro, Y. E. M. (2019b) Etnobotânica de Parahancornia fasciculata (Apocynaceae): extração, usos e comercialização do leite de amapá na comunidade da ilha Trambioca, Barcarena, Pará, Brasil. Scientia Plena, 15 (11), 1-8. 10.14808/sci.plena.2019.112701.

De Sousa, R. L., Miranda, A. U. S., Cordeiro, Y. E. M., Pereira, M. G. (2019a). Extração e comercialização do óleo de andiroba (Carapa guianensis Aublet.) na comunidade da Ilha das Onças, no município de Barcarena, Pará, Brasil. INTERAÇÕES, Campo Grande, MS, 20 (3), 879-889, http://dx.doi.org/10.20435/inter.v0i0.1826.

Dorneles, L. N. S., Gonelia, A. L. D., Cardoso, C. A. L., Silva, C. B., Hautha, M. R., Oba, G. C., Schoeninger, V. (2019). Effect of air temperature and velocity on drying kinetics and essential oil composition of Piper umbellatum L. leaves. Industrial Crops \& Products, 142 (2019), 111846. https://doi.org/10.1016/j.indcrop.2019.111846.

Emmanuel, A., Daniela, U., Marielba, M., Luis, R-F., Juan, C. (2018). Estudio de la composición química de los aceites esenciales de las hojas y flores de Leonotis nepetifolia (L.) R. Br. (Lamiaceae). Revista de la Facultad de Farmacia, 60 (2), 25-30. http://www.saber.ula.ve/handle/123456789/46325.

Ferreira, O. O., Cruz, J. N., Franco, C. J. P., Silva, S. G., Costa, W. A., Oliveira, M. S., Andrade, E. H. A. (2020). First Report on Yield and Chemical Composition of Essential Oil Extracted from Myrcia eximia DC (Myrtaceae) from the Brazilian Amazon. Molecules, 25, 783. 10.3390/molecules25040783.

Filho, J. L. S. C., Blank, A. F., Alves, P. B., Ehlert, P. A. D., Melo, A. S., Cavalcanti, S. C.H., Arrigoni-Blank, M. F., Silva-Mann, R. (2006). Influence of the harvesting time, temperature and drying period on basil (Ocimum basilicum L.) essential oil. Revista Brasileira de Farmacognosia, 16 (1), 24-30. http://www.sbfgnosia.org.br/revista/

Fonseca, D. J. S., Neto, J. P. A., Costa, J. M. (2018). Banho de Cheiro de São João no município de Abaetetuba, Pará, Brasil. Revista África e Africanidades, Ano XI - 27, jul. ISSN: 1983-2354. https://africaeafricanidades.net/index.html

Freitas, A. V. L., Coelho, M. F. B., Pereira, Y. B., Freitas Neto, E. C., Azevedo, R. A. B. (2015). Diversidade e usos de plantas medicinais nos quintais da comunidade de São João da Várzea em Mossoró, RN. Revista Brasileira de Plantas Medicinais, Campinas, 17 (4), 845-856. 10.1590/1983-084x/14_080.

Leal, J. B., Silva, M. M., Costa, J. M., Albuquerque, L. C. S., Pereira, M. G. S., Sousa, R. L. (2019). Etnobotânica de plantas medicinais com potencial aintiinflamatório utilizadas pelos moradores de duas comunidades no município de Abaetetuba, Pará. Biodiversidade, 18 (3), 110-125. https://periodicoscientificos.ufmt.br/ojs/index.php/biodiversidade/about.

Lima, R. K., \& Cardoso, M. G. (2007). Família Lamiaceae: Importantes Óleos Essenciais com ação biológica e antioxidante. Revista Fitos, 3 (03), 14-24. $10.32712 / 2446-4775.2020 .826$.

Lopes, C. B., Camara, C. A. G., Moraes, M. M. (2019). Composition of essential oils from the leaves, stems, and flowers of Vernonia condensata of Pernambuco, Brasil. Chemistry of Natural Compounds, 55 (4), 756. 10.1007/s10600-019-02802-8.

Maurya, A. K., Bahl, J. R., Yadav, A., Prakash, O., Saxena, A., Pal, A., Chanotiya, C. S., Khan, F., Bawankule, D. U. (2019). Chemical composition and skin inflammation protective profile of pulgone rich essential oil of Mentha arvensis L. India Journal of Experimental Biology, 57, 168-174. http://nopr.niscair.res.in/handle/123456789/55803.

Mesquita, S. S., Cruz, A. S., Leal, J. B., Sousa, R. L. (2020). Etnobotânica das plantas medicinais utilizadas nos cuidados com a saúde na comunidade nossa Senhora do Livramento, Acará, Pará. Gaia Scientia, 14 (2), 142-159. https://doi.org/10.22478/ufpb.1981-1268.2020v14n3.51133.

Mesquita, U. O., Tavares-Martins, A. C. C. (2018). Etnobotánica de plantas medicinales en la comunidad de Caruarú, Isla del Mosqueiro, Belém-PA, Brasil. Boletín Latinoamericano y del Caribe de Plantas Medicinales y Aromáticas, 17 (2), 130-159. https://doi.org/10.37360/blacpma.21.20.1.1.

Meyer, L., Quadros, K. E., Zeni, A. L. B. (2012). Etnobotânica na comunidade de Santa Bárbara, Ascurra, Santa Catarina, Brasil. Revista Brasileira Biociências, 10 (3), 258-266. http://www.ufrgs.br/seerbio/ojs/index.php/rbb/article/view/4119.

Ming, L. C. Coleta de plantas medicinais. In: DI Stasi, L. C. (Org.). Plantas Medicinais: Arte e Ciência - Um Guia de Estudo Interdisciplinar. Editora da Universidade Estadual Paulista, 1996.

Mohamad, H., Andriani, Andriani, Y., Bakar, K., Siang, C. C., Syamsumir D. F., Alias, A., Radzi, S. A. M. (2015). Effect of drying method on anti-microbial, anti-oxidant activities and isolation of bioactive compounds from Peperomia pellucida (L) Hbk. Journal of Chemical and Pharmaceutical Research, 7 (8), 578-584. http://www.jocpr.com.

Neri, G., Oliveira, T. L., Oliveira, V. J. S., Brito, N. M. (2018). Uso de plantas medicinais nas unidades de Saúde da família do Alto Sobradinho e Cocão do município de Santo Antônio de Jesus-BA. Ensaios Ciencias, 22 (1), 58-62. 10.17921/1415-6938.

Noriega, P., Guerrini, A., Sacchetti, G., Grandini, A., Ankuash, E., Manfredini, S. (2019). Chemical Composition and Biological Activity of Five Essential Oils from the Ecuadorian Amazon Rain Forest. Molecules, 24 (8), 1637. 10.3390/molecules24081637.

Okoh, S. O., Iweriebor, B. C., Okoh, O. O., Okoh, A. I. (2017). Bioactive constituents, radical scavenging, and antibacterial properties of the leaves and stem essential oils from Peperomia pellucida (L.) Kunth. Pharmacognosy Magazine, 13, S392-400. 10.4103/pm.pm_106_17. 
Oliveira, J. S. F., Xavier, L. P., Lins, A., Andrade, E. H., Maia, J. G. S., Mello, A. H., Setzer, W. N., Ramos, A. R., Silva, J.K. (2019a). Effects of inoculation by arbuscular mycorrhizal fungi on the composition of the essential oil, plant growth, and lipoxygenase activity of Piper aduncum L. AMB Express, 9 (1), 29. 10.1186/s13568-019-0756-y.

Oliveira, S. S., Hanada, R. E., \& Brito, R. S. (2019b). Composição química e atividade antifúngica do óleo essencial de Zingiber officinale Roscoe sobre Colletotrichum theobromicola, causador da antracnose da cebolinha (Allium fistulosum). Scientia Naturalis, 1 (1): 32-40. http://revistas.ufac.br/revista/index.php/SciNat.

Orlanda, J. F. F., \& Nascimento, A. R. (2015). Chemical composition and antibacterial activity of Ruta graviolens L. (Rutaceae) volatile oils, from São Luís, Maranhão, Brasil. South African Journal of Botany, 99, 103-106. http://dx.doi.org/10.1016/j.sajb.2015.03.198.

Pereira, M. G. S., \& Coelho-Ferreira, M. (2017). Uso e diversidade de plantas medicinais em uma comunidade quilombola na Amazônia Oriental, Abaetetuba, Pará. Biota Amazônia, 7 (3), 57-68. http:/ dx.doi.org/10.18561/2179-5746/biotaamazonia.v7n3p57-68.

Pinto, L. N., Flor, A. S. S. O., \& Barbosa, W. L. R. (2014). Fitoterapia popular na Amazônia Paraense: uma abordagem no município de Igarapé-Miri, estado do Pará nos anos de 2000 e 2008. Revista Ciências Farmacêuticas Básica e Aplicada, 35 (2), 305-311. https://doi.org/10.4322/2179-443X.0636

Quinlan, M. (2005). Considerations for collecting freelists in the field: examples from Ethobotany. Field Methods, 17 (3), 1-16. https://journals.sagepub.com/home/fmx.

Rodrigues, F. F. G., Costa, J. G. M., Rodrigues, F. F. G., \& Campos, A. R. (2013). Study of the Interference between Plectranthus Species Essential Oils from Brazil and Aminoglycosides. Evidence-Based Complementary and Alternative Medicine, 2013, 1-7. http://dx.doi.org/10.1155/2013/724161.

Santos, E. Q., Costa, J. F. S., Pereira, M. G. S., Costa, J. M., Sousa, R. L. (2019). Etnobotânica da flora medicinal de quintais na comunidade Mamangal, Igarapé-Miri, PA. Scientia Plena, 15 (5), 1-11. 10.14808/sci.plena.2019.051202.

Silva, J. K. R., Silva, N. N. S., Santana, J. F. S., Andrade, E. H. A., Maia, J. G. S., \& Setzer, W. N. (2016). Phenylpropanoid-rich essential oils of Piper species from the Amazon and their antifungal and anti-cholinesterase activities. Natural Product Communications, 11 (12), 1907-19011. $10.1177 / 1934578 X 20985222$.

Silva, N. C., Gonçalves, S. F., Araújo, L. S., Kasper, A. A. M., Fonseca, A. L, Sartoratto, A., Castro, K. C. F., Moraes, T. M. P., Barattos, L. C., Varotti, F. P., Barata, L. E. S., Moraes, W. P. (2019b). In vitro and in vivo antimalarial activity of the volatile oil of Cyperus articulatus (Cyperaceae). Acta Amazonica, 49 (4), 334-342. http://dx.doi.org/10.1590/1809-4392201804331.

Silva, S. G., Costa, R. A. C., Oliveira. M. S., Cruz, J. N., Figueiredo, P. L. B., Brasil, D. S. B., Nascimento, L. D., Neto, A. M. J. C., Junior, R. N. C., Andrade, E. H. A. (2019a) Chemical profile of Lippia thymoides, evaluation of the acetylcholinesterase inhibitory activity of its essential oil, and molecular docking and molecular dynamics simulations. PLoS ONE, 14 (3), e0213393. https://doi.org/10.1371/journal. pone.0213393.

Silvestre, W. P., Medeiros, F. R., Agostini, F., Toss, D., Pauletti, G. F. (2019). Fractionation of rosemary (Rosmarinus officinalis L.) essential oil using vacuum fractional distillation. Journal of Food Science Technology, 56, 542-5434. https://doi.org/10.1007/s13197-019-04013-z.

Simonian, L. T. L. Ilha Trambioca através da história: recursos, ocupações humanas e cultura. In: Simonian, L.T.L (Org.). Gestão em ilha de muitos recursos, história e hábitos: experiências na Trambioca (Barcarena, PA). Belém: NAEA-UFPA; Projeto NAEA/Fundação Ford, 2004. 73-136.

Soares, A. S., Pastores, J. F. B., Jardim, J. G. (2019). Lamiaceae no Rio Grande do Norte, Brasil. Rodriguésia, 70, e03312017. http://dx.doi.org/10.1590/21757860201970067.

Souihi, M., Amri, I., Souissi, A., Hosni, K., Brahim, N. B., Annabi, M. (2020). Essential oil and fatty acid composition of Melissa officinalis L. Progress in Nutrition, 22 (1): 253-258. 10.23751/pn.v22i1.7758.

Souza, A. P. S. \& Lisboa, R. C. L. (2005). Musgos (Bryophyta) na Ilha Trambioca, Barcarena, PA, Brasil1. Acta Botanica Brasilica, 19 (3), $487-492$.

Souza, T. A., Lopes, M. B. P., Ramos, A. S., Ferreira, J. L. P., Silva, J. R. A., Queiroz, M. M. C., Araújo, K. G. L., Amaral, A. C. F. (2018). Alpinia essential oils and their major components against Rhodnius nasutus, a vector of Chagas Disease. The Scientific World Journal, 2018, 1-6. https://doi.org/10.1155/2018/2393858.

Verma, R. S., Padalia, R. C., Goswami, P., Chauhan, A. (2014). Essential oil composition of Peperomia pellucida (L.) Kunth from India. Journal of Essential Oil Research, 16 (15), 1-6. http://dx.doi.org/10.1080/10412905.2014.982878.

Vila, R., Mundina, M., Tomi, F., Furlán, R., Zacchino, S., Casanova, J., Cañigueral, S. (2001). Composition and antifungal activity of the essential oil of Solidago chilensis. Planta Medica, 68, 164-167. 10.1055/s-2002-20253.

Vilhena, C. S., Nascimento, L. A. S., Andrade, E. H. A., Silva, J. K. R., Hamoyc, M., Torres, M. F., Barbas, L. A. L. (2019). Essential oil of Piper divaricatum induces a general anaesthesia-like state and loss of skeletal muscle tonus in juvenile tambaqui, Colossoma macropomum. Aquaculture, 510, 169-175. https://doi.org/10.1016/j.aquaculture.2019.05.057.

Violante, I. M. P., Garceza, W. S., Barbosa, C. S., Garceza, F. R. (2012). Chemical composition and biological activities of essential oil from Hyptis crenata growing in the Brazilian Cerrado. Natural Product Communications, 7 (10), 1387-1389. 10.1177/1934578X20985222.

Wakabayashi, K. A. L., Melo, N. I., Aguiar, G. P., Carvalho, C. E., Ramos, R. C., Mantovani, A. L. L., Groppo Jr, M., Veneziani, R. C. S., Crotti, A. E. M. (2010). Chemical composition of the essential oil from the leaves of Alternanthera brasiliana (L.) Kuntze (Amaranthaceae). Investigação,10, 82-5. https://doi.org/10.26843/investigacaov1922020.

Zoghbi, M. D. G., Andrade, E. H., Carreira, L. M., Rocha, E. A. (2008). Comparison of the main components of the essential oils of “priprioca": Cyperus articulatus var. articulatus L., C. articulatus var. nodosus L., C. prolixus Kunth and C. rotundus L. Journal of Essential Oil Research, 20 , 42-45. https://doi.org/10.1080/10412905.2020.1804001. 